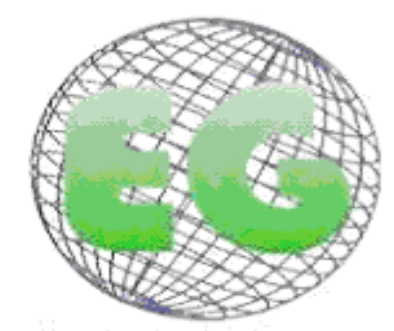

ISSN 1695-6141 N 27

\title{
La alimentación preescolar, educación para la salud de los 2 a los 6 años
}

The pre-school feeding, health education from 2 to 6 years

\section{${ }^{*}$ Cubero J, *Cañada $\mathrm{F},{ }^{*}$ Costillo $\mathrm{E},{ }^{* *}$ Franco $\mathrm{L}$, " Calderón $\mathrm{A},{ }^{* * *}$ Santos $\mathrm{AL}$, ${ }^{* * *}$ Padez C, *Ruiz C.}

\begin{abstract}
*Laboratorio de Educación para Salud. Área de Dca de las Ciencias Experimentales. Facultad de Educación. Universidad de Extremadura. Badajoz. E-mail: jcubero@unex.es **Grupo de Investigación: Neuroinmunofisiología y Crononutrición. Dpto. de Fisiología. Facultad de Ciencias. Universidad de Extremadura. ${ }^{* * *}$ Dpto. de Ciencia de la Vida. Facultad de Ciencia y Tecnología. Universidad de Coimbra. Portugal.
\end{abstract}

Palabras clave: alimentación infantil; nutrición; educación; salud. Keywords: Infant feeding; nutrition; education; health.

\section{RESUMEN}

La alimentación y nutrición infantil constituye una de las áreas de mayor importancia dentro de la Educación para la Salud (EpS). Esta acción promotora de la salud debe iniciarse en la fase temprana del desarrollo y aprendizaje, como es la etapa preescolar. Una valiosa herramienta para iniciar la EpS es la alimentación infantil, la cual puede y debe ser abordada en el ámbito educativo.

Por ello, nuestro objetivo de investigación fue recoger, de los principales manuales de nutrición españoles, las características básicas, nutricionales, de la etapa de 2 a 6 años, para ser utilizado por personal docente no sanitario en el ámbito escolar. Mediante análisis de revisión bibliográfica documentada, referente a las características nutricionales de esta etapa de crecimiento.

Los resultados indican que las Recomendaciones Diarias Alimenticias (RDA) a partir de los 2 años son muy diferentes respecto a la etapa de lactante, el aporte se balancea hacía los carbohidratos en un $50 \%$, en decremento de grasas $<30 \%$ y proteínas. Además, la preparación e incorporación de nuevos ingredientes a los platos diarios es ya una constante, obteniendo nuevas y atractivas características organolépticas para conseguir una dieta equilibrada y saludable. Para ello, se seguirá una pauta de 3 comidas, con 2 intermedias de menor cantidad, y donde el desayuno ya tendrá una merecida importancia.

Para concluir, además debemos indicar que el educador a través de la EpS debe trasmitir e inculcar, desde esta temprana edad, hábitos de vida saludable a través de la alimentación infantil. Ya que esta dieta infantil será la base de una etapa adulta saludable, pues es en la etapa infantil donde se fraguan futuras patologías como diabetes, cáncer de colon e hipertensión. 


\section{ABSTRACT}

The infant feeding and nutrition is one of the most important areas regarding the Health Education (HE). This health-promoting action must start at the early stage of the development and learning, such as the pre-school period. A valuable tool to initiate successfully the HE could be infant nutrition, which can and must be also approached in the educational area.

Our objective of the investigation was to gather, from the main Spanish manuals of nutrition, the basic and nutritional characteristics of the stage from 2 to 6 years, in order to be used by educational personnel instead of health personnel in the school area. By the analysis of bibliographical documented review, related to the nutritional characteristics at this stage of growth.

The results indicate that the Recommendations Dietary Allowance (RDA) from 2 years are very different with regard to the breast-fed stage, the contribution moves towards the carbohydrates in $50 \%$, in decline of fats $<30$ $\%$ and proteins. In addition, the preparation and incorporation of new ingredients to the daily foods is already regular, obtaining, in this way, a balanced and healthy diet. For that, we will follow a guideline of 3 daily foods, with 2 intermediate of minor quantity, and the breakfast will have a well-deserved importance.

To conclude, we must indicate that the educator has to inculcate healthy habits of feeding in pre-school stage by means of the Health Education. The mentioned infant diet will be the base for a healthy adulthood, since during the infant stage, future pathologies may emerge such as diabetes, cancer of colon and hypertension.

\section{INTRODUCCIÓN}

La alimentación y nutrición infantil comprende una de las áreas de mayor importancia dentro de la Educación para Salud (EpS), disciplina relativa al área de la Salud Pública, la cual trabaja para educar en el cuidado de la salud, potenciando hábitos de vida saludable. A través de EpS se trata de enseñar estilos de vida saludable, y es en la etapa infantil cuando más se puede y debe incidir sobre la tarea del fomento de la salud así como la prevención de enfermedades tanto infantiles, como en la etapa posterior de la adolescencia y por último en la madurez ${ }^{[1,2]}$. Por lo tanto dentro EpS, la alimentación infantil en esta etapa de crecimiento, es una herramienta indispensable para la promoción de la salud.

En el segundo año de vida, transcurridos los primeros 4-6 meses donde sus requerimientos nutricionales han sido aportados mediante lactancia exclusiva, $y$ posteriormente tras un año de una alimentación complementaria o de beikost ${ }^{[3,4,5,6]}$, se llega a la etapa preescolar, que según Cobaleda y Bousoño se inicia a los 2 años. Dicha etapa del crecimiento engloba el periodo desde que el niño adquiere autonomía en la marcha locomotora hasta que inicia el periodo escolar, es decir los 6 años ${ }^{[3]}$. Aunque otros autores ${ }^{[7]}$ describen esta etapa de preescolar a niños que abarca de entre 4 y 6 años de edad que comienza por el periodo de crecimiento estable y un progresivo desarrollo psicomotor y social. Indicar que a su vez, también, esta etapa de crecimiento puede quedar dividida desde el ámbito educativo: en un primer ciclo de 0 a 3 años seguido de en un segundo ciclo de 3 a 6 años.

\section{OBJETIVO}

Por lo descrito, nuestro objetivo de investigación fue recopilar de los principales manuales de nutrición españoles, las características básicas nutricionales y dietéticas de en la etapa de 2 a 6 años. Para ser usado por personal docente no sanitario, pero con reconocida labor promotora de salud a nivel escolar, y así favorecer el fomento de la EpS en el ámbito de la nutrición y alimentación infantil. 


\section{METODOLOGÍA}

Mediante análisis de revisión bibliográfica documentada, de los manuales editados por las principales sociedades médicas y de nutrición españolas, referente a las Requerimientos nutricionales; macronutrientes y micronutriente. Así como las Recomendaciones dietéticas, para esta etapa de crecimiento infantil de 2 a 6 años.

\section{RESULTADOS}

\section{Requerimientos nutricionales}

Está comprobado científicamente que la alimentación extrauterina e infantil independiente del paso al nacer, influye en las anormalidades metabólicas en la etapa adulta ${ }^{[3,8]}$.

\section{Energía}

Las necesidades calóricas bajan, pero las proteicas, sin embargo, aumentan por el crecimiento de los músculos y otros tejidos. Es preciso tener en cuenta que las recomendaciones se basan en estimaciones donde la edad es la principal variable aglutinadora pero existen otros variables como las antropométricas, además del entorno sociocultural en que nos encontremos, y que no son tomadas en cuenta.

Por ello, las necesidades energéticas serán individualizadas y dependerán del gasto calórico demandado por el crecimiento, el metabolismo basal de las funciones fisiológicas y la actividad física. Incidiendo en que esta es una etapa de crecimiento, donde la fase de anabolismo es trascendental, y se demanda una gran cantidad de energía.

Siguiendo las indicaciones del Comité de Nutrición de la Asociación Española de Pediatría recogidas en el Manual Práctico de Nutrición Pediátrica ${ }^{[3]}$ podemos indicar que las Recomendaciones Diarias de Administración (RDA) para un niño de 2 a 3 años se estiman desde $1.000 \mathrm{Kcal}$ si es sedentario a 1.400 si es activo.

\section{Hidratos de Carbono}

Su aporte, en esta etapa de crecimiento, debe aumentan entorno al 50-55\% como base energética (4 kcal/ g), en decremento de las grasas. Sus necesidades, para ambos sexos, serán por tanto $130 \mathrm{~g} / \mathrm{día}$.

Es conveniente que los azúcares simples mono y disacáridos (glucosa, fructosa y sacarosa) no superen el 10\%. El 90\% restante de los carbohidratos serán complejos y por lo tanto de absorción lenta (almidón y fibra dietética) aumentado por ello el consumo de cereales, legumbres, verduras, hortalizas y frutas. Está comprobado científicamente que una temprana y regulada ingesta de todos estos últimos alimentos servirá para disminuir el índice glicémico sanguíneo, previniendo con ello enfermedades crónicas en la etapa madurez como son: diabetes, obesidad, hipertensión, cáncer de colón, enfermedades cardiovasculares $\left.{ }^{[8,9}\right]$.

\section{Proteínas}

Los requerimientos para este macronutriente en la etapa de preescolar es de entre 10-15\% de las calorías (4 Kcal/g de proteína). Inicialmente de los 1 a 3 años esta debe de ser de 1,1 g/kg/día (13 g/día), disminuyendo a 0,95 g/kg/día hasta la adolescencia (34 g/día). 
Respecto a las proteínas de origen animal indicar que la albúmina de huevo es el alimento de mayor calidad proteica seguida de la caseína de la leche, ya que contienen la mayor cantidad y calidad de aminoácidos esenciales; treonina, metionina, lisina, valina, leucina, isoleucina y fenilalanina, sin olvidar el valioso triptófano ${ }^{[10]}$. Además del aminoácido histidina que para esta etapa de desarrollo está considerado como aminoácido esencial.

Respecto a las proteínas de origen vegetal (cereales y legumbres) indicar que son de menor calidad proteica que las de origen animal pero son ricas, respectivamente, en metionina (precursor de la cisteína) y lisina.

\section{Grasas}

A partir de los 2 años de vida (nunca antes) se recomienda reducir la ingesta total de grasas hasta un mínimo de $30 \%$ del total de calorías, ya que dietas con muy bajo contenido en grasas están asociadas a un retraso en el crecimiento ${ }^{[11,12]}$.

Aparte de energía (9 kcal/g), las grasas también nos van aportar vitaminas liposolubles, ácidos grasos esenciales y colesterol.

La distribución debe ser un $10 \%$ para grasas saturadas (de origen animal; mantecas y de origen vegetal: margarinas y mantequillas, que sin son de origen animal tendrá un potencial aterogénico, por ello no se sobrepasará el porcentaje mencionado. Pero no podemos olvidar, que también nos aportará el valioso colesterol, (aporte máximo <300 mg/día), precursor de hormonas esteroídeas, vitaminas y ácidos biliares, sin olvidar que también aporta plasticidad a todas la membranas celulares.

Otro $10 \%$ será para las valiosas grasas monoinsaturadas, como es el caso del acido oleico, presente en nuestro aceite de oliva, base de la saludable dieta mediterránea.

El 10\% restante será para las grasas poliinsaturadas (PUFA), ambos grupos son fuente de los famosos ácidos grasos de cadena larga (precursores de protaglandinas, tromboxanos y leucotrienos, partículas que previenen enfermedades cardiovasculares) como es el caso del linoléico ( $\square 6)$ presente en huevos y aceites vegetales cuyo aporte recomendado es de entre $1-2 \%$, y por último el linolénico $(\square 3)$ presentes en el pescado azul, aunque no podemos olvidar que dicho contenido de linolénico depende de la estación de año, en que sea realizada la captura para la sardina, atún, caballa, salmón.

\section{Vitaminas}

Como fuente de Vitaminas Liposolubles (K, A, D, E) se debe fomentar el consumo de germen de cereales, hortalizas y verduras de hoja verde, los aceites de origen vegetal, los productos lácteos no desnatados y yema de huevo en particular para la vitamina $\mathrm{E}$.

Respecto a las Vitaminas Hidrosolubles (Complejos B y C) las encontramos principalmente en el germen de cereales e hígado. Así como en particular en la yema de huevo para la vitamina B7 y en los cítricos para la, antioxidante, vitamina $\mathrm{C}$.

\section{Minerales}

Divididos en dos tipos: Macroelementos y Elementos trazas. 


\section{-Macroelementos}

Calcio: las recomendaciones diarias hasta los 3 años de vida corresponden a $500 \mathrm{mg}$, y de los 3 a los 8 años los requerimientos diarios se incrementan hasta 800 mg. Será principalmente a través de una ingesta de $500 \mathrm{ml}$ de leche o sus derivados, el alimento que nos aportará este mineral necesario para cubrir el crecimiento óseo.

Fósforo: sus necesidades diarias serán similares a las del calcio, de 450-500 mg/día.

Electrolitos: Sodio el estudio de los aportes en el Proyecto STRIP ${ }^{[13]}$, indican una valoración a los 3 años de edad de $1.900 \mathrm{mg} \pm 527 \mathrm{mg}$, indicando que a medida que iba aumentado la edad había aumentando el consumo de sal de mesa. En cuanto al Magnesio las RDA entre 1 y 3 años sería de $80 \mathrm{mg} /$ día y entre 4 y 8 años $130 \mathrm{mg} /$ día. Los alimentos ricos en magnesio son las legumbres, chocolate, y cereales.

\section{-Elementos traza}

Divididos, a su vez, en otros dos tipos: Microelementos (RDA: mg/día) y Elementos trazas (RDA: $\square \mathrm{g} / \mathrm{día}$ ).

\section{--Microelementos}

Hierro: sus requerimientos rondan entre $7-10 \mathrm{mg} / \mathrm{día}$. Como alimentos recomendados fuente de este mineral se aconseja la ingesta de carne, huevo, legumbres y cereales. Indicar que el hierro de cereales y legumbres se absorbe peor y esta absorción puede mejorar si conjuntamente se ingiere vitamina C.

Zinc: las recomendaciones diarias en esta etapa de crecimiento rondan los $10 \mathrm{mg}$. Dicho mineral es necesario para el anabolismo muscular lo encontramos principalmente en carne roja.

Flúor: sus requerimientos están entre 0,9 y $1 \mathrm{mg} / \mathrm{día}$, su aporte vendrá a través del agua. Sus depósitos serán esmalte y hueso.

Cobre: las recomendaciones diarias serán superiores a $0,1 \mathrm{mg}$. Esencial para mineralización del esqueleto, sistema nervioso, inmune, coagulación y metabolismo de la glucosa y el colesterol.

\section{--Oligoelementos}

Yodo: los requerimientos diarios serán de $200 \square \mathrm{g}$ de 1-3 años, y de $300 \square \mathrm{g}$ de 4-8 años. Su aporte se realizará a través de la sal común yodada.

Selenio: los requerimientos diarios serán de $20 \square \mathrm{g}$ de 1-3 años, y de $30 \square \mathrm{g}$ de 4-8 años, con función antioxidante y que se puede encontrar en las carnes rojas.

\section{Recomendaciones dietéticas}

Siguiendo, principalmente, las pautas del AEP [3] y de la SENC [15] se recomienda: 
Practicar un correcto desayuno: levantar al niño con tiempo suficiente para desayunar, con toda la familia, de una forma tranquila y sosegada, es decir utilizar para ello el tiempo estimado (15-20 minutos).

Si el niño no está habituado a desayunar se comenzará al principio con un vaso de leche o un yogur. Luego se incorporará algún tipo de cereal como tostadas, galletas maría o cereales para desayuno y finalmente una fruta o zumo natural. En ocasiones podemos dividir el desayuno en dos tomas, una al levantarnos con leche o yogur, cereales, pan o galletas, y la otra dos o tres horas después donde podemos incorporar la fruta.

Es recomendable que los niños consuman diariamente entre medio y un litro de leche u otros lácteos.

Iniciemos, progresivamente, en la dieta de los niños la valiosa norma de 5 raciones al día de frutas y verduras.

Moderar el consumo de carnes en los niños, especialmente procesadas y en embutidos y reducir la ingesta de azucares simples, dulces y bollería industrial, aumentando por ello su ingesta en carbohidratos complejos: pan, pasta, legumbres, patatas y cereales.

Cuidar la preparación de los alimentos: higiene, textura y características organolépticas; color, olor, sabor y flavor. Ya que plato bien elaborado estimula las ganas de comer y despierta el interés del niño.

Servir las raciones apropiadas en tamaño según edad y gasto energético.

Recordar que los niños de 2 a 3 años de edad necesitan el mismo número de raciones que los de 4 a 6 años, pero serán más pequeñas (2/3 de la ración).

Establecer horarios regulares así como un ambiente agradable y distendido.

Enseñarlo a comer solo, dedicando el tiempo necesario, así como a colaborar en poner la mesa.

Complementar la cena con la comida del mediodía, sobre todo en los niños que usan comedores escolares.

Por último y no menos importantes:

No permitir el juego ni ver la televisión mientras se come.

Evitar el abuso de bollería industrial, golosinas y refrescos.

Recordar que el niño es el que regula la cantidad de lo que come, según la sensación de hambre o saciedad. No se debe forzar, ni amenazar y menos manifestar ansiedad en el momento de las comidas.

En resumen, dietéticamente se aconseja seguir nuestra famosa y saludable Dieta Mediterránea Tradicional, pero adaptada a las recomendaciones diarias, para esta etapa de crecimiento. Tal y como se indica en las siguientes Pirámides de Alimentación Figura 1 y 2., Advertir que en las etapas de crecimiento infantil así como en la adolescencia, no existirá 
ningún tipo de ingesta de alcohol. Y las raciones diarias para cada grupo de alimentos, serán adaptadas a las exigencias, antropométricas y de edad.

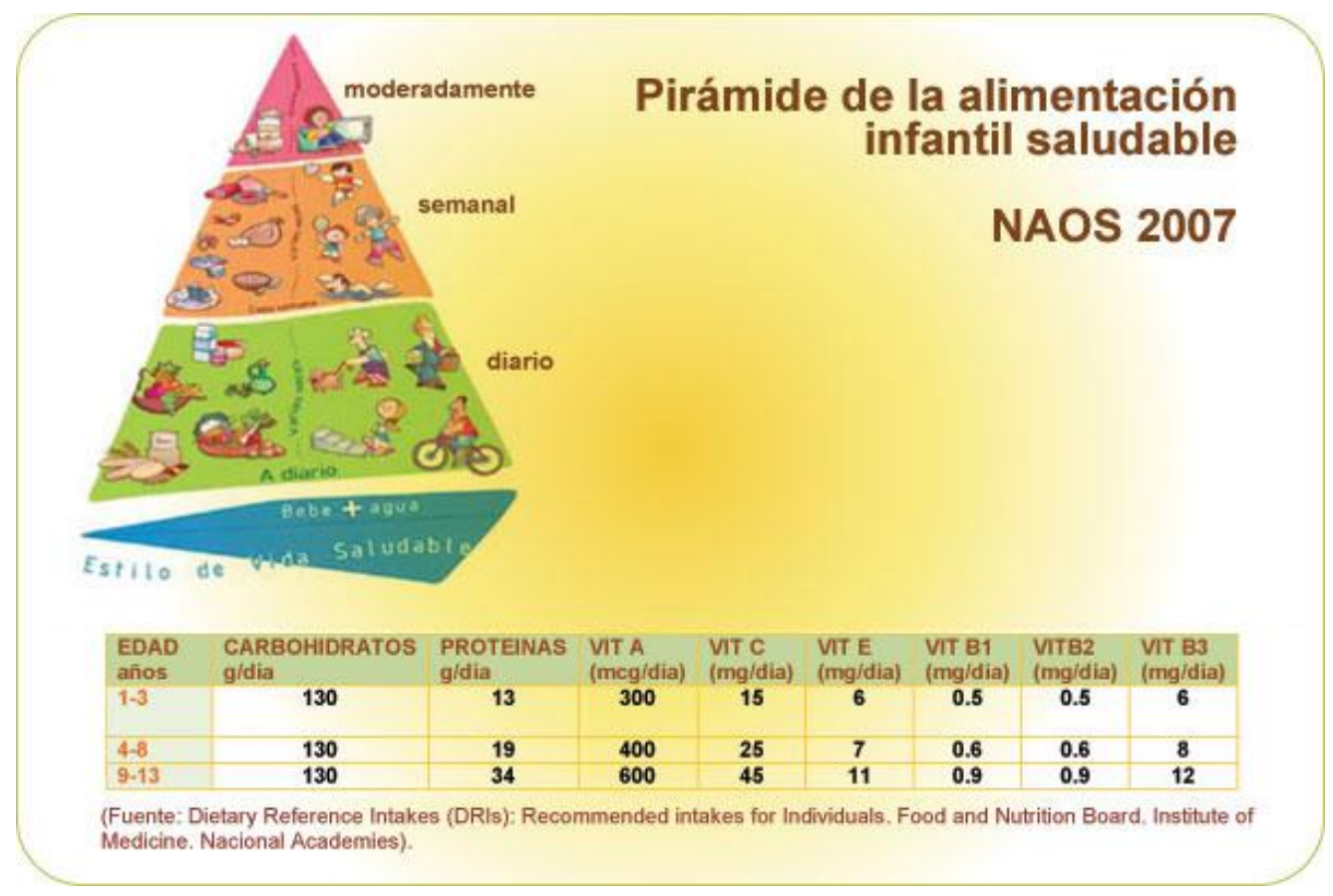

Figura 1. Pirámide de la alimentación saludable. Estrategia para la nutrición la actividad física y la prevención de la Obesidad. Estrategia NAOS. http://www.naos.aesan.msps.es/ ${ }^{[14]}$.

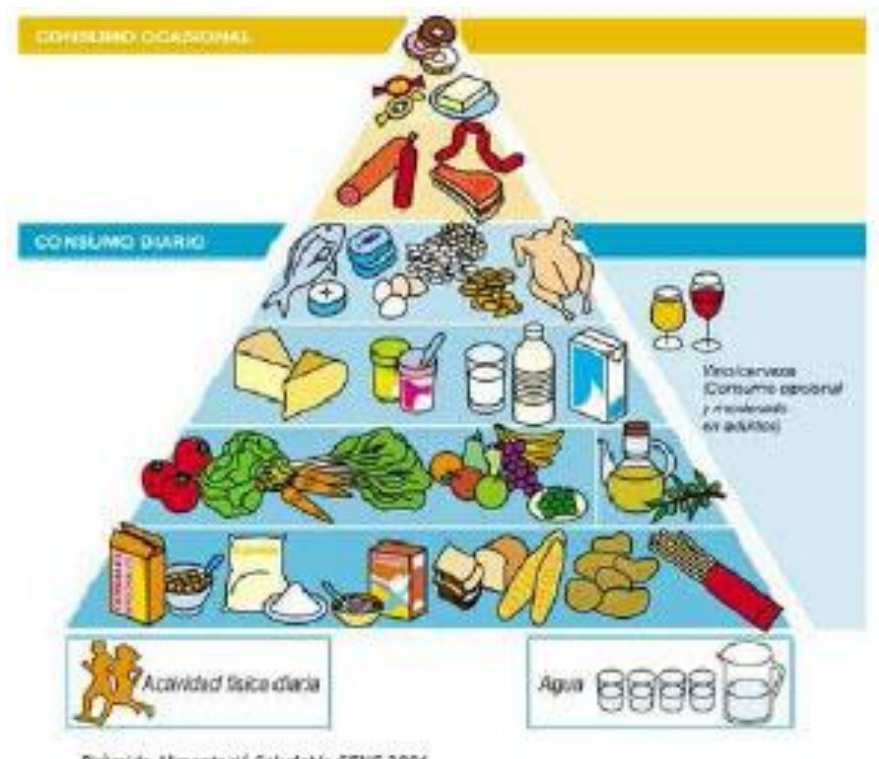

Figura 2. Pirámide de la Alimentación Saludable de la Sociedad Española de Nutrición Comunitaria (SENC). http://www.nutricioncomunitaria.org/index.jsp ${ }^{[15]}$. 


\section{El papel de la familia y los educadores, en la alimentación preescolar}

Sería un error pensar que todo lo que los alumnos aprenden forma parte del currículo, hay otras formas de aprendizaje no escritas, entre ellas la imitación, y en esta edad sus dos modelos de identidad fundamentales son sus padres y sus profesores. Hay otros ámbitos de influencia que no podemos desdeñar su importancia como son los medios audiovisuales, en este campo de la alimentación.

Los niños aprenden por imitación, por ello el papel de los padres y maestros en la educación alimentaria es determinante, también influirán en él sus compañeros y amigos, la publicidad en los medios de comunicación, en particular la televisión y TICs.

A través de EpS se trata que los alumnos adopten estilos de vida saludable, y es en esta etapa infantil cuando más se puede y se debe incidir en dicha disciplina, debido a las características de maleabilidad y aceptación de nuevos patrones de conducta.

Padres y educadores deben crear unos hábitos alimentarios saludables durante la infancia, contribuyendo a un correcto crecimiento y desarrollo de los niños, lo cual es fundamental para evitar enfermedades crónicas en la edad adulta, "Se come en función de lo que comen los mayores" ${ }^{[3]}$, y tras nuestra reflexión añadiríamos: “... y yermiten los mayores".

En base a ello se demanda una formación, universitaria, del personal docente en EpS, con contenidos firmes en alimentación y nutrición infantil. La cual no solo debería estar presente, principalmente, en el currículum de grado de maestro de infantil, sino en el de etapas posteriores como primaria y resto de grados y máster relacionados con la educación y ciencias de la salud, dentro del Espacio Europeo de Educación Superior [16].

Porque si desde la infancia se adoptan y promocionan buenos hábitos alimentarios a través de la EpS, estos favorecerán la práctica de un estilo de vida más saludable en la edad adulta, previniendo patologías como las conocidas obesidad, diabetes, hipertensión, enfermedad cardiovascular y cáncer.

\section{CONCLUSIONES}

Para finalizar podemos concluir, indicando que las Recomendaciones Diarias Alimenticias (RDA) a partir de los 2 años son muy diferentes respecto a la etapa de lactante, el aporte se balancea hacía los carbohidratos en un $50 \%$, en decremento de grasas $<30 \%$ y proteínas. Además, la preparación e incorporación de nuevos ingredientes a los platos diarios es ya una constante, obteniendo nuevas y atractivas características organolépticas, para conseguir una dieta equilibrada y saludable. Para ello, se seguirá una pauta de 3 comidas, con 2 intermedias de menor cantidad, y donde el desayuno ya tendrá una merecida importancia. Además debemos indicar que el educador a través de la EpS debe trasmitir e inculcar, desde esta temprana edad, hábitos de vida saludable a través de la alimentación infantil. Ya que esta dieta infantil será la base de una etapa adulta saludable, pue es en la etapa infantil donde se fraguan futuras patologías como diabetes, cáncer de colon e hipertensión.

\section{AGRADECIMIENTOS}

Al Plan I.D.T.I de la UEx, 2010. Acción VII. Proyectos de Iniciación a la Investigación y el Desarrollo Tecnológico. Consejería de Sanidad y Dependencia. Junta de Extremadura. Vicerrectorado de Investigación, Innovación e Infraestructura Científica. Universidad de Extremadura. 


\section{BIBLIOGRAFÍA}

[1] Serrano M.I.. La educación para la Salud del Siglo XXI. Comunicación y Salud. 1997.Madrid: Ediciones Díaz de Santos.

[2] Ruíz C, Calderón MA, Bas MA. Formación de Formadores en Educación para la Salud. 2006. Merida: Ediciones Junta de Extremadura. Consejería de Sanidad y Consumo.

[3] Suarez L. Manual práctico de Nutrición en Pediatría. 2007. Comité de Nutrición de la AEP. Ediciones Ergón. Madrid.

[4] Peña L. Alimentación del preescolar y escolar. Protocolos diagnósticos y terapéuticos en pediatría. 2001. 321-328.

[5] Ballabriga A, Carrascosa A. Nutrición en la Infancia y en la adolescencia. 2001. $2^{\mathrm{a}}$ Ed. Ergon S.A. Madrid

[6] Sánchez C, Narciso D, Rivero M, Sánchez S, Johnston S, Sánchez J, Rodríguez $A B$, Cubero J. Nociones en la alimentación y nutrición infantil durante el primer año de vida. Enfermería Global. 2008. 8.

[7] Gómez C, Cos A. Nutrición en atención primaria. 2001. Jarpyo Editores. Madrid.

[8] González-Barranco J, Ríos-Torres JM. Early malnutrition and metabolic abnormalities later in life. Nutr Rev. 2004. 62. 134-139.

[9] Yanik CS. The lifecycle effects of nutrition and body size on adult adiposity, diabetes and cardiovascular disease. Obes Rev. 2002. 3(3):217-24.

[10] Cubero J, A. B. Rodríguez, D. Narciso, V. Valero, J. Sánchez, C. Barriga. Anotaciones básicas del aminoácido triptófano. Enfermería Global. 2006. 12

[11] Uauy R, Mize CE, Castillo-Duran C. Fat intake during childhood: metabolic responses and effects on growth. Am J Clin Nutr. 2000. 72. 135-136.

[12] Hardy SC, Kleinman RE. Fat and cholesterol in the diet of infants and young children: implications for growth, development, and long-term health. J Pediatr. 1994. 125. 69-77.

[13] Lagström H, Jokinen E, Seppanen R. Nutrient intakes by young by young children in a prospective randomized trial of low-saturated trial of low-saturated fat, low cholesterol diet. The STRIP Baby Project. Arch Pedatr Adol Med. 1997. 151:181-8.

[14] La alimentación de tus niños/as. Estrategia NAOS. Agencia Española de Seguridad Alimentaria y Nutrición (AESAN). 2010. Ministerio de Sanidad, Política Social e Igualdad. SENC.

[15] Guía de la alimentación saludable. SENC. Serra Majem L. 2004. Ediciones

[16] Cubero J, Costillo E, Calderón M, Ruiz C. La Educación para la Salud en el Espacio Europeo de Educación Superior. Publicaciones. 2011. Bajo revisión.

ISSN 1695-6141

๑ COPYRIGHT Servicio de Publicaciones - Universidad de Murcia 